\title{
Risk of total/subtotal meniscectomy for respective medial and lateral meniscus injury: correlation with tear type, duration of complaint, age, gender and ACL rupture in 6034 Asian patients
}

Dong Jiang ${ }^{1}$, Xiao Luo ${ }^{1}$, Yingfang Ao ${ }^{1}$, Xi Gong ${ }^{1}$, Yong-jian Wang ${ }^{1}$, Hai-jun Wang ${ }^{1}$, Yu Miao ${ }^{1}$, Nan Li², Ji-ying Zhang ${ }^{1}$ and Jia-kuo $\mathrm{Yu}^{\text {}^{*}}$

\begin{abstract}
Background: To evaluate the risk factor associated with total or subtotal meniscectomy for respective medial and lateral meniscus injury.

Methods: The data of all the meniscus injured patients undergoing arthroscopy in our institute between January 15th, 2000 and December 31st, 2008 was collected and 6034 patients with 7241 injured menisci met the inclusion criteria. The mean patient age was $33.6 \pm 14.9$ years and there were 4785 males and 2456 females with 3568 medial and 3673 lateral menisci. The decision tree approach was applied to investigate the correlation of the tear type, the duration of complaint, age, gender, ACL rupture and total/subtotal meniscectomy for respective medial and lateral meniscus.
\end{abstract}

Results: The tear type was associated with both medial $(x 2=70.901, P<0.001)$ and lateral $(x 2=268.019, P<0.001)$ total/subtotal meniscectomy. The strongest risk of total/subtotal meniscectomy of both medial and lateral meniscus tear was shown for the complex tear followed by the longitudinal, oblique, horizontal and radial tear of the medial meniscus and followed by horizontal, longitudinal, radial and oblique tear of the lateral meniscus. The risk of total/ subtotal medial meniscectomy was significantly elevated for the patients with complex tear and the age of $\leq 40$ years old $(X 2=21.028, P<0.001)$ and those with the oblique, horizontal or radial tear accompanied by ACL rupture $(X 2=6$. $631, P=0.01)$. Besides, the duration of complaint was also associated with total/subtotal meniscectomy of the medial longitudinal tear with $A C L$ rupture $(X 2=17.155, P<0.001)$. On the other side, the risk of total/subtotal lateral meniscectomy was significantly elevated for the complex tear of the female patients $(x 2=5.877, P=0.015)$ with no $A C L$ rupture $(X 2=50.501, P<0.001)$. The $A C L$ rupture was associated with a decreased risk of total/subtotal meniscectomy for all the types of the lateral meniscus (complex: $X 2=50.501, P<0.001$; horizontal: $X 2=20.897$, $P<0.001$; oblique: $x 2=27.413, P<0.001$; longitudinal and radial: $X 2=110.85, P<0.001$ ).

(Continued on next page)

\footnotetext{
*Correspondence: yujiakuo@126.com

Dong Jiang and Xiao Luo are co-first authors.

Dong Jiang and Xiao Luo are equally contributed to this work.

'Institute of Sports Medicine, Peking University Third Hospital, Beijing Key

Laboratory of Sports Injuries, Beijing 100191, China

Full list of author information is available at the end of the article
} 
(Continued from previous page)

Conclusion: Analyzing data from a big sample available in an Asian patient database, we found different risk factors associated with total/subtotal meniscectomy for respective medial and lateral meniscus. Identifying patients at high risk for total/subtotal meniscectomy may allow for interventions after meniscus injury.

Keywords: Meniscectomy, Meniscus injury, Medial, Lateral, Risk factor

\section{Background}

Meniscus injury is one of the most common sports injuries $[1,2]$. As a vital part of the knee joint, the meniscus serves important roles including load bearing, shock absorption, stabilization, lubrication, nutrition of the articular cartilage and proprioception [3, 4]. Despite of meniscus repair and transplantation, total or subtotal meniscectomy was still inevitable for severe meniscus injury. Biomechanical studies have confirmed the decrease in intra-articular contact area and increase in the peak contact pressure after meniscectomy $[5,6]$, especially after total or subtotal meniscectomy, which significantly elevated the risk of osteoarthritis [7]. Thus identifying patients at high risk for total/subtotal meniscectomy may allow for interventions after meniscus injury.

Some risk factors have been confirmed to be associated with meniscus injuries $[8,9]$, including gender, sport, and type of exposure [5, 10, 11]. A large series of patients undergoing anterior cruciate ligament (ACL) reconstruction identified that increased age, male gender, and increased surgical delay were associated with a higher frequency and severity of meniscus injuries [12]. However, the correlation between the surgical option (partial or total meniscectomy or repair) and the patients' parameters has been rarely studied.

In addition, the difference have been confirmed to exist in the injury characteristics between the medial and lateral meniscus [13, 14]. Dandy et al. [13] investigated 1000 symptomatic meniscus injury patients and found that there were significantly more medial meniscus tears in males than in females, there were more longitudinal tears than horizontal tears in the medial meniscus, and the most common tear type of the lateral meniscus is a longitudinal tear. Bergkvist [15] et al. reported 1375 cases of degenerative meniscal tears in 4096 arthroscopies, finding that there were more posterior horn tears in medial radial tears and more meniscal body tears in lateral radial tears. The difference of medial and lateral menisci in tear characteristics might affect the surgical option, especially the total/subtotal meniscectomy. So far, few study has evaluated the risk factor correlated with the total/subtotal meniscectomy, especially comparing the difference between medial and lateral meniscus.

The purpose of the present study was to evaluate the risk factor associated with total or subtotal meniscectomy for respective medial and lateral meniscus injury through the analysis of the 7241 meniscal tears. We hypothesized that the tear type, the duration of complaint, age, gender and ACL rupture might be associated with the risk of total/subtotal meniscectomy and the difference might exist between the medial and the lateral meniscus.

\section{Methods}

\section{General information}

The study was a retrospective study and was approved by the Medical Ethics Committee of Peking university third hospital (No: IRB00006761-2011097). A total of 6736 patients underwent arthroscopic meniscus treatment in our institute between January 15th, 2000 and December 31st, 2008. Those patients with discoid meniscus injuries, posterior cruciate ligament tears or collateral ligament tears were excluded thus 6034 patients with 7241 torn menisci were included in this study. The mean patient age was $33.6 \pm 14.9$ years and there were 4785 males and 2458 females with 3568 medial and 3673 lateral menisci.

There were 4037 patients diagnosed with ACL rupture and underwent ACL reconstruction at the time of meniscus surgery. The duration of complaint was recorded from the primary injury or the symptom onset of the patients with not clear trauma history. For detailed statistical analysis, the duration of complaint were divided into 0-28 days, 29-56 days, 57 day- 1 year, 1-2 year and $>2$ years. In addition, the patients were also divided into 2 groups based on age $\leq 40$ years and $>40$ years.

All patients were given spinal/epidural anesthesia with bupivacaine. All the surgeries were performed with arthroscopy (Smith-Nephew, Boston, MA, USA) according to a unified and standardized protocol. The joint was thoroughly examined by anteromedial and anterolateral portal using the 30 degree scope in sequential manner. The meniscal tear was confirmed by probing with the same principle of operation indication and technique referenced to the O'Connor's classification [16]. The tear types of both medial and lateral meniscus was recorded as longitudinal, horizontal, oblique, radial, and complex. The surgeons removed all ruptured and offending meniscal tissue and left a stable and smooth rim of meniscus. When the degenerative changes and ruptures were present within the entire meniscus and the rupture 
reached as far as the synovial junction, total meniscectomy was performed. Excision of the pathological tissue was carried out with punch and mechanical shaver. In most of the cases partial meniscectomy and repair were kept as the first choice of treatment, preferable to subtotal or total meniscectomy. The meniscectomy was recorded as partial if any part of the meniscus was removed, leaving a minimum of two-thirds of the meniscal surface intact. More than one-third of the meniscal surface resection was recorded as subtotal $[17,18]$, most of which underwent posterior horn and mid-third resection with an intact rim and root attachments. All the data including the parameters of the patients and the management of all the meniscus tears were obtained from the medical records.

\section{Data analysis and statistics}

All the data were analyzed using SPSS software (version 24.0; IBM Corporation, Armonk, New York). Two data sets were built from the source data set. Medial meniscus injured set involved patients with the diagnosis of medial meniscus tears while the lateral meniscus injured set involved patients with the diagnosis of lateral meniscus tears. For each set, the total or subtotal meniscectomy were treated as outcome events, while the partial meniscectomy or meniscus repair were considered as not event. Then the age was translated into binary variable of $\leq 40$ years or $>40$ years; while the time to operation was translated into categorical variable. For the univariate analysis, all categorical variable such as age over 40, time slot to operation, gender, side, ACL injured were described with proportion and compared with Chisquare test.

Since the tear category was complicated, and interaction might exist between multiple variables. In order to make the results easier to interpret, the decision tree approach was applied to investigate the association between these factors and total/subtotal meniscectomy. The decision tree was set up by SPSS 24 and established by CHAID method. The minimum number of father and child nodes was 100 and 50, respectively, and the maximum tree depth is 3 . A $P$ value of less than 0.05 was considered statistically significant.

\section{Results}

There were 1124 (31.5\%) medial and 1413 (38.5\%) lateral meniscus tears undergoing total/subtotal meniscectomies respectively with significant difference $\left(\chi^{2}=15.47\right.$, $P<0.001)$. Besides, there were 1621 (45.5\%) medial and 1937 (52.7\%) lateral meniscus tears undergoing partial meniscectomies respectively with significant difference ( $x 2=37.754, P<0.001$ ). Significant more repairs of medial meniscus were performed than those of lateral meniscus $\left(730(20.5 \%)\right.$ vs $\left.323(8.8 \%), \chi^{2}=198.194, P<0.001\right)$.
The general information and the univariate analysis of the respective medial and lateral meniscus tears were shown in Table 1. The Chi-square test showed significant difference in the age, the duration of complaint and the tear type for both medial and lateral meniscus $(P<0.05)$. No significant difference was found in the injured side for both medial and lateral meniscus (n.s.).

The percentage of total/subtotal meniscectomy for the patients $\leq 40$ was more than those $>40$ for the medial meniscus tears while the opposite results were found for the lateral meniscus. Compared to the male patients with lateral meniscus tear, the total/subtotal meniscectomy was prone to the female patients with lateral meniscus tear $\left(\chi^{2}=23.595, \quad P<0.001\right)$. No significant difference was found between medial meniscus injured patients accompanied by or without ACL rupture (n.s.) while more total or subtotal meniscectomy was found in the lateral meniscus injured patients with intact ACL than those with ACL rupture $\left(X^{2}=248.09, P<0.001\right)$.

The results of the decision tree for the medial meniscus tear was shown in Fig. 1 and the gains of nodes were shown in Table 2. The tear type was associated with medial total/subtotal meniscectomy $(\chi 2=70.901$, $P<0.001)$. The strongest risk of total/subtotal meniscectomy was shown for the complex tear (39.9\%) followed by the longitudinal (31\%) and oblique, horizontal and radial tear (22.2\%). The risk of total/subtotal medial meniscectomy was significantly elevated for the complex tear with less than 40 years old $\left(x^{2}=21.028, P<0.001\right)$ and the oblique, horizontal or radial tear accompanied by $\mathrm{ACL}$ rupture $\left(x^{2}=6.631, P=0.01\right)$. Besides, the duration of complaint was associated with total/subtotal meniscectomy of the medial longitudinal tear accompanied by ACL rupture ( $\mathrm{x} 2=17.741, P=0.004)$.

The results of the decision tree for the lateral meniscus tear was shown in Fig. 2 and the gains of nodes were shown in Table 3. The tear type was also associated with the lateral total/subtotal meniscectomy $\left(\chi^{2}=268.019, P<0.001\right)$. The strongest risk of total/subtotal meniscectomy was shown for the complex tear $(54.6 \%)$ followed by the horizontal (38.9\%) and the longitudinal, radial $(31.2 \%)$ and oblique tear $(16.7 \%)$. The risk of total/subtotal lateral meniscectomy was significantly elevated for the complex tear of the female patients $\left(\chi^{2}=5.877, P=0.015\right)$ with no ACL rupture $\left(X^{2}=50.501, P<0.001\right)$. The risk of total $/$ subtotal lateral meniscectomy was significantly elevated for patients with the oblique tear accompanied by ACL rupture in the left side $\left(\chi^{2}=7.609, P=0.006\right)$. The ACL rupture was associated with a decreased risk of total/subtotal meniscectomy for all the types of the lateral meniscus (complex: $\chi^{2}=50.501, P<0.001$; horizontal: $\chi^{2}=$ 20.897, $P<0.001$; oblique: $\chi^{2}=27.413, P<0.001$; longitudinal and radial: $\left.X^{2}=110.85, P<0.001\right)$. 
Table 1 General Information and univariate analysis of Medial and Lateral Meniscus Tears

\begin{tabular}{|c|c|c|c|c|c|}
\hline \multirow[b]{2}{*}{ Variable } & \multirow[b]{2}{*}{ Groups } & \multicolumn{2}{|l|}{ Medial } & \multicolumn{2}{|l|}{ Lateral } \\
\hline & & Meniscus tears & Total/subtotal meniscectomy (\%) & Meniscus tears & Total/subtotal meniscectomy (\%) \\
\hline \multirow[t]{3}{*}{ Age } & $\leq 40$ & 2525 & $825(32.7 \%)$ & 2891 & 1056(36.5\%) \\
\hline & $>40$ & 1043 & $299(28.7 \%)$ & 782 & $357(45.7 \%)$ \\
\hline & $x 2 / P$ & & $x^{2}=5.489, P=0.019^{*}$ & & $x 2=21.652, P<0.001^{*}$ \\
\hline \multirow[t]{3}{*}{ Gender } & Female & 1243 & $391(31.5 \%)$ & 1213 & $534(44 \%)$ \\
\hline & Male & 2325 & $733(31.5 \%)$ & 2460 & $879(35.7 \%)$ \\
\hline & $x 2 / P$ & & $X 2=0.002, P=0.965$ & & $X 2=23.595, P<0.001^{*}$ \\
\hline \multirow[t]{3}{*}{ Side } & Left & 1734 & $523(30.2 \%)$ & 1712 & $654(38.2 \%)$ \\
\hline & Right & 1834 & $601(32.8 \%)$ & 1961 & 759(38.7\%) \\
\hline & $\times 2 / P$ & & $X 2=2.81, P=0.094$ & & $x^{2}=0.098, P=0.754$ \\
\hline \multirow[t]{3}{*}{$\mathrm{ACL}$} & Intact & 1480 & $441(29.8 \%)$ & 1724 & $895(51.9 \%)$ \\
\hline & Rupture & 2088 & $683(32.7 \%)$ & 1949 & $518(26.6 \%)$ \\
\hline & $x 2 / P$ & & $X 2=3.407, P=0.065$ & & $X 2=248.09, P<0.001^{*}$ \\
\hline \multirow[t]{6}{*}{ Duration of complaint } & $0-28$ days & 310 & $93(30 \%)$ & 404 & $131(32.4 \%)$ \\
\hline & 29-56 weeks & 247 & $58(23.5 \%)$ & 285 & $90(31.6 \%)$ \\
\hline & $57 d-1$ year & 1680 & $513(30.57 \%)$ & 1773 & 675(38.1\%) \\
\hline & $1-2$ year & 462 & $149(32.3 \%)$ & 439 & $170(38.7 \%)$ \\
\hline & $>2$ years & 869 & $311(31.5 \%)$ & 772 & $347(44.9 \%)$ \\
\hline & $x 2 / P$ & & $X 2=15.933, P=0.003^{*}$ & & $X 2=25.771, P<0.001^{*}$ \\
\hline \multirow[t]{6}{*}{ Tear types } & Longitudinal & 1624 & $504(31 \%)$ & 826 & $272(32.9 \%)$ \\
\hline & Radial & 219 & $55(25.1 \%)$ & 585 & $168(28.7 \%)$ \\
\hline & Oblique & 253 & $57(22.5 \%)$ & 480 & $80(16.7 \%)$ \\
\hline & Horizontal & 408 & $83(20.3 \%)$ & 507 & 197(38.9\%) \\
\hline & Complex & 1064 & $425(39.9 \%)$ & 1275 & $696(54.6 \%)$ \\
\hline & $x 2 / P$ & & $x 2=72.426, P<0.001^{*}$ & & $x^{2}=270.585, P<0.001^{*}$ \\
\hline
\end{tabular}

*represents the significant difference $(P<0.05)$

\section{Discussion}

Current meniscus repair techniques are only effective in treating lesions located in the peripheral vascularized region of the meniscus. Thus total or subtotal meniscectomy was still inevitable for the severe meniscus tears. Identifying patients at high risk for total/subtotal meniscectomy may allow for interventions after meniscus injury. However, few study has evaluated the risk factor associated with the total/ subtotal meniscectomy, especially comparing the difference between medial and lateral meniscus. The purpose of the present study was to evaluate the risk factor of total or subtotal meniscectomy for respective medial and lateral meniscus injury. Analyzing data from a big sample available in an Asian patient database, we found different risk factors associated with total/subtotal meniscectomy for respective medial and lateral side. According to the results, meniscus tears should be treated as soon as possible to reduce the risk of total or subtotal meniscectomy, especially for the medial complex tear with age of $\leq 40$, other medial tear type companied by ACL rupture and the lateral complex tear of female patients with intact ACL.
The tear type was significantly associated with both medial and lateral total/subtotal meniscectomy. The strongest risk of total/subtotal meniscectomy of both medial and lateral meniscus tear was shown for the complex tear followed by the longitudinal, oblique, horizontal and radial tear of the medial meniscus and followed by horizontal, longitudinal, radial and oblique tear of the lateral meniscus. As a special tear type, the complex tear usually combined with variety of tear types including oblique, horizontal and longitudinal tears, which significantly increased its risk of total/subtotal meniscectomy. The difference in the strength of the risk for the other tear types might be due to the difference of the anatomy and the dynamics between the medial and the lateral meniscus. Compared to the other tear types, the stability of the medial meniscus would be obviously decreased with the longitudinal tear, which was associated high risk of total/subtotal meniscectomy. On the other hand, there were 3 popliteomeniscal fascicles, which combined with the popliteus tendon form a peripheral hooplike attachment to the lateral meniscus but 


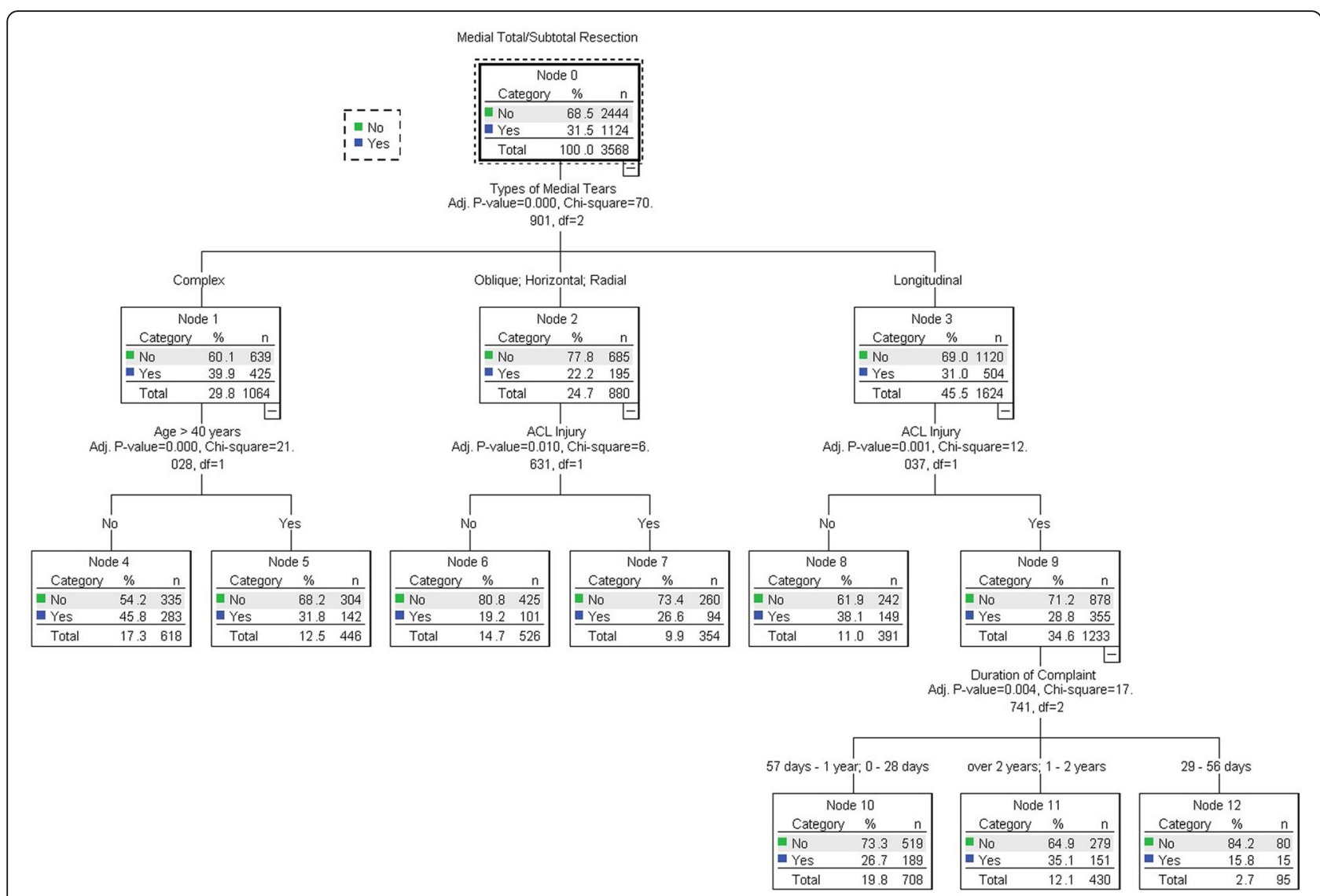

Fig. 1 Results of the decision tree for the medial meniscus tear. The tear type was associated with medial total/subtotal meniscectomy $(x 2=70.901$, $P<0.001)$. The strongest risk of total/subtotal meniscectomy was shown for the complex tear (39.9\%) followed by the longitudinal (31\%) and oblique, horizontal and radial tear (22.2\%). The risk of total/subtotal medial meniscectomy was significantly elevated for the complex tear with less than 40 years old $(X 2=21.028, P<0.001)$ and the oblique, horizontal or radial tear accompanied by ACL rupture $(X 2=6.631, P=0.01)$. Besides, the duration of complaint was associated with total/subtotal meniscectomy of the medial longitudinal tear accompanied by ACL rupture $(X 2=17.741, P=0.004)$

not to the medial meniscus. The popliteus complex provided both a static and dynamic connection between the lateral meniscus and the popliteus tendon and controlled the motion of the lateral meniscus during flexion and extension of the knee [19-22]. It was difficult to reconstruct both the static and dynamic connection for the

Table 2 Gains for Nodes of medial meniscus tear

\begin{tabular}{|c|c|c|c|c|c|c|}
\hline \multirow[t]{2}{*}{ Node } & \multicolumn{2}{|c|}{ Node } & \multicolumn{2}{|l|}{ Gain } & \multirow[t]{2}{*}{ Response } & \multirow[t]{2}{*}{ Index } \\
\hline & $\bar{N}$ & $\overline{\text { Percent }}$ & $\overline{\mathrm{N}}$ & $\overline{\text { Percent }}$ & & \\
\hline 4 & 618 & $17.30 \%$ & 283 & $25.20 \%$ & $45.80 \%$ & $145.40 \%$ \\
\hline 8 & 391 & $11.00 \%$ & 149 & $13.30 \%$ & $38.10 \%$ & $121.00 \%$ \\
\hline 11 & 430 & $12.10 \%$ & 151 & $13.40 \%$ & $35.10 \%$ & $111.50 \%$ \\
\hline 5 & 446 & $12.50 \%$ & 142 & $12.60 \%$ & $31.80 \%$ & $101.10 \%$ \\
\hline 10 & 708 & $19.80 \%$ & 189 & $16.80 \%$ & $26.70 \%$ & $84.70 \%$ \\
\hline 7 & 354 & $9.90 \%$ & 94 & $8.40 \%$ & $26.60 \%$ & $84.30 \%$ \\
\hline 6 & 526 & $14.70 \%$ & 101 & $9.00 \%$ & $19.20 \%$ & $61.00 \%$ \\
\hline 12 & 95 & $2.70 \%$ & 15 & $1.30 \%$ & $15.80 \%$ & $50.10 \%$ \\
\hline
\end{tabular}

lateral horizontal tear by meniscus repair. Thus the risk of total/subtotal meniscectomy was significant elevated.

Our study demonstrated another interesting result that increasing age was associated with a decreased risk of medial total/subtotal meniscectomy while the risk was elevated for the patients of $\leq 40$ with complex tear. Previous studies have showed that increased age predicted intra-articular injuries [23-25]. However, few of them discussed about the correlation between increased age and the risk of meniscectomy. Kluczynski et al. [23] found that increased age predicted more meniscectomies in males. Ralles [26] et al. concluded that age was an independent predictor for medial meniscus injury. The discrepant findings from these researches might be due to the different data which include only the patients underwent ACL reconstructions. The present study included the patients both with and without ACL rupture. The protective effect of age on medial meniscus tears is probably due to the decrease in physical activity level after meniscus tears. In addition, a considerable proportion of the meniscus tears in elder patients were due to 


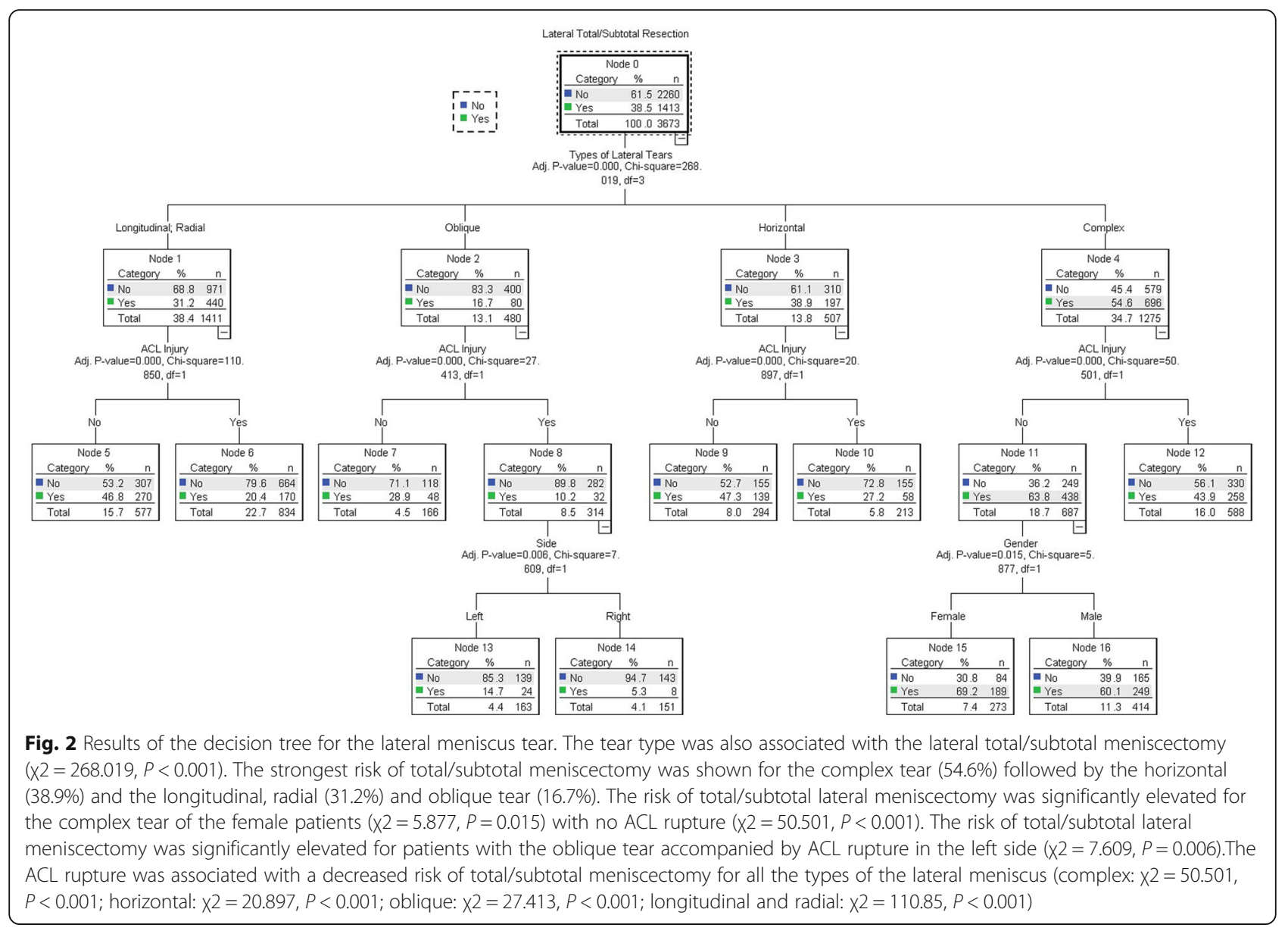

degeneration and the horizontal tear was usually only located at the posterior horn of medial meniscus. Thus partial meniscectomy was kept as the first choice other than total/subtotal meniscectomy for those patients, which might decrease the risk of total/subtotal meniscectomy.

As the most common associated injury, ACL rupture lead to abnormal joint forces and are associated with

Table 3 Gains for Nodes of lateral meniscus tear

\begin{tabular}{|c|c|c|c|c|c|c|}
\hline \multirow[t]{2}{*}{ Node } & \multicolumn{2}{|c|}{ Node } & \multicolumn{2}{|l|}{ Gain } & \multirow[t]{2}{*}{ Response } & \multirow[t]{2}{*}{ Index } \\
\hline & $\bar{N}$ & $\overline{\text { Percent }}$ & $\overline{\mathrm{N}}$ & Percent & & \\
\hline 15 & 273 & $7.40 \%$ & 189 & $13.40 \%$ & $69.20 \%$ & $180.00 \%$ \\
\hline 16 & 414 & $11.30 \%$ & 249 & $17.60 \%$ & $60.10 \%$ & $156.30 \%$ \\
\hline 9 & 294 & $8.00 \%$ & 139 & $9.80 \%$ & $47.30 \%$ & $122.90 \%$ \\
\hline 5 & 577 & $15.70 \%$ & 270 & $19.10 \%$ & $46.80 \%$ & $121.60 \%$ \\
\hline 12 & 588 & $16.00 \%$ & 258 & $18.30 \%$ & $43.90 \%$ & $114.10 \%$ \\
\hline 7 & 166 & $4.50 \%$ & 48 & $3.40 \%$ & $28.90 \%$ & $75.20 \%$ \\
\hline 10 & 213 & $5.80 \%$ & 58 & $4.10 \%$ & $27.20 \%$ & $70.80 \%$ \\
\hline 6 & 834 & $22.70 \%$ & 170 & $12.00 \%$ & $20.40 \%$ & $53.00 \%$ \\
\hline 13 & 163 & $4.40 \%$ & 24 & $1.70 \%$ & $14.70 \%$ & $38.30 \%$ \\
\hline 14 & 151 & $4.10 \%$ & 8 & $0.60 \%$ & $5.30 \%$ & $13.80 \%$ \\
\hline
\end{tabular}

increased risk of injury to menisci, which normally transmit load and absorb shocks between the femur and tibia [27]. Our results concluded the idea of a significant decrease in the percentage of lateral total/subtotal meniscectomies with ACL rupture. This seems contradictory from the widely accepted view that the risk of meniscus tear and meniscectomy would be increased by the instability of ACL ruptured joint. A systematic review summarized data from 159 studies over the last 10 years which showed that meniscectomy was the most common method of treatment, performed overall in $65 \%$ of the meniscus tears [28]. Hagino et al. [29] reported lower percentage of meniscectomy, with $39 \%$ to $55 \%$ based on different surgical-timing group. However, Bellabarba et al. [30] summarized that the likelihood of a successful meniscal repair is enhanced significantly when combined with ACL reconstruction. Intra-articular bleeding caused by ACL reconstruction is also considered to provide a favorable environment for meniscal healing [31]. Thus total/subtotal meniscectomy would be decreased owing to the meniscus repair. Another reason of the risk reduction might be due to less sports activities with pain, giving way and loss of range of motion after ACL rupture, which partly prevented the existing 
meniscus tears from developing to a serious type. Compared to the isolated meniscus tears, those with concurrent ACL rupture experienced a completely different mechanical environment of the knee joint, which might affect the risk of total/subtotal meniscectomy. Further studies are needed in the future.

According to the results, the ACL rupture was confirmed to be a protective factor for the lateral meniscectomy but be a risk factor for the medial meniscectomy. The reason might be due to the difference of the biomechanics and the anatomy between medial and lateral meniscus. For one thing, the medial meniscus is less mobile than the lateral meniscus. Thus it is less suitable to follow the increased anterior and rotational translation of the tibia, especially into dynamic situations [24, 32]. Feucht [33] et al. demonstrated that with ACL injury, male patients, patients $<30$ years and patients who sustained a contact injury had a high risk of major lateral meniscal tear. Arner [34] et al. reported greater translation with ACL deficiency in lateral meniscus compared with medial meniscus by using minimally invasive techniques that may explain the greater incidences of acute lateral meniscus tears and chronic medial meniscus tears. In the present study, most of the cases was chronic meniscus tears with the duration of complaint of more than 1 months. Thus the aggravation of the meniscus tear was prone to the medial meniscus. For another, the tears near the hiatus could produce a small and unstable meniscal bridge and the stripe could dislocate into the joint space and cause incarceration [35]. The incarcerated meniscus would severely destroy the normal force transmission structure of the knee and result in further damage. Thus the lateral meniscus tear worsen faster than the medial meniscus tear in the ACL intact knee.

In terms of the duration of complaint, the difference also existed between respective medial and lateral side. The duration of complaint was not associated with the lateral total/subtotal meniscectomy. However, the risk of total or subtotal meniscectomy increased significantly for the patients with medial longitudinal tear accompanied by ACL rupture. The reason might be due to the mechanical environment changes after ACL rupture, especially for the longitudinal tear as the most unstable meniscus injury. Previous studies have shown that lateral meniscal tears are commonly associated with acute ACL tears, while medial meniscal tears are often associated with chronic ACL tears [36, 37]. Bramilla et al. [32] selected 1069 consecutive patients who underwent primary ACL reconstruction, recommended that the reconstruction not be delayed more than a year after the injury, owing to the increased risk of medial meniscal tears. Ralles [26] et al. illustrated that patients who received surgery $>12$ months after initial injury underwent stronger risk of injury in medial meniscus than those within 12 months. Chhadia et al. [24], who chose similar time ranges, showed an increase of medial meniscus tears at 6 months from the injury. They also found a strong association of prolonged time to surgery with decreased meniscus repair rate. Krutsch [38] et al. made a recommendation for an ACL reconstruction within 6 months after trauma to preserve the medial meniscus from resection, which is consistent with our results. However, all the above studies were performed for the meniscus patients with concurrent ACL rupture, which was also one of the import factors affecting the treatment option. In the present study, all the meniscus tear patients with or without ACL rupture were included. The results was parallel to the above studies but further distinguished the difference between the medial and the lateral meniscus.

To our knowledge, this is the first study evaluating the risk factors of total/subtotal meniscectomy for respective medial and lateral meniscus. In spite that the trend of prevalence of meniscus tears was well studied by many previous research, including gender, sport, and type of exposure $[5,10,11]$, few study analyzed about surgical options and time-window about meniscus lesions, especially for respective medial and lateral side. The results of the present study offer insight into the correlation of those factors with total/subtotal meniscectomy for respective medial and lateral meniscus and was beneficial for the surgeon and the patient more focusing on the surgical strategy to preserve meniscus as much as possible.

There were still some limitations for the present study. First, the level of evidence was low due to the single center retrospective study. Despite being one of the largest sports medicine centers of the country, the differences in patients' sources, diagnosis and treatment principle between institutes might affect the results. Besides, the meniscus tear type have not be classified by widely accepted classifications by Warren [39] or West [40] and the root tear (10 $\mathrm{mm}$ from the insertion site) have not be distinguished but was only recorded as the radial or oblique tear of the posterior horn before 2000. The meniscus root tear might be analyzed according to the data after 2010 with future studies. Furthermore, the inaccurate course of the disease might affect the results since the duration of complaint was used instead of the injury duration. Approximately one third patients suffered meniscus tear but without any trauma history and those meniscus tears might be due to some small unnoticed trauma or the degeneration.

\section{Conclusion}

Analyzing data from a big sample available in an Asian patient database, we found different risk factors associated with total/subtotal meniscectomy for respective 
medial and lateral meniscus. Identifying patients at high risk for total/subtotal meniscectomy may allow for interventions after meniscus injury. Meniscus tears should be treated as soon as possible to reduce the risk of total or subtotal meniscectomy, especially for the medial complex tear with age of $\leq 40$, other medial tear type accompanied by ACL rupture and the lateral complex tear of female patients with intact ACL.

\section{Abbreviation}

$\mathrm{ACL}$ : Anterior cruciate ligament

\section{Acknowledgements}

None.

\section{Funding}

The study was funded by the National Natural Scientific Foundation of China (Grant No. 81630056, 31670982).

\section{Availability of data and materials}

The medical records are kept in the department of medical records of our hospital and it is not publicly available due to it may compromise the anonymity of the patients. However, the raw data will be available from the corresponding author upon reasonable request.

\section{Authors' contributions}

JD and LX wrote and modified the manuscript and did the data analysis. YA participated in its design and coordination. GX, WYJ, WHJ and MY helped in data collecting and coordination. LN helped to do the data statistics. ZJY helped in wring and editing the manuscript. YJK conceived the study and helped to draft the manuscript. All authors read and approved the final manuscript.

\section{Ethics approval and consent to participate}

The study was approved by the Medical Ethics Committee of Peking university third hospital (No: IRB00006761-2011097).

\section{Consent for publication}

No details, images, or videos relating to an individual person were included in the manuscript.

\section{Competing interests}

The authors declare that they have no competing interests.

\section{Publisher's Note}

Springer Nature remains neutral with regard to jurisdictional claims in published maps and institutional affiliations.

\section{Author details}

${ }^{1}$ Institute of Sports Medicine, Peking University Third Hospital, Beijing Key Laboratory of Sports Injuries, Beijing 100191, China. ${ }^{2}$ Research Center of Clinical Epidemiology, Peking University Third Hospital, No.49 North Garden Road, Haidian, Beijing 100191, China.

Received: 25 March 2017 Accepted: 21 November 2017

Published online: 05 December 2017

\section{References}

1. Abrams GD, Frank RM, Gupta AK, Harris JD, McCormick FM, Cole BJ. Trends in meniscus repair and meniscectomy in the United States, 2005-2011. Am J Sports Med. 2013;41(10):2333-9.

2. Ferry $T$, Bergstrom U, Hedstrom EM, Lorentzon R, Zeisig E. Epidemiology of acute knee injuries seen at the emergency Department at Umea University Hospital, Sweden, during 15 years. Knee Surg Sports Traumatol Arthrosc. 2014;22(5):1149-55.

3. Mordecai SC, Al-Hadithy N, Ware HE, Gupte CM. Treatment of meniscal tears: an evidence based approach. World J Orthop. 2014;5(3):233-41.
4. Krause WR, Pope MH, Johnson RJ, Wilder DG. Mechanical changes in the knee after meniscectomy. J Bone Joint Surg Am. 1976;58(5):599-604.

5. Baratz ME, Fu FH, Mengato R. Meniscal tears: the effect of meniscectomy and of repair on intraarticular contact areas and stress in the human knee. A preliminary report. Am J Sports Med. 1986;14(4):270-5.

6. Ahmed AM, Burke DL. In-vitro measurement of static pressure distribution in synovial joints-part I: Tibial surface of the knee. J Biomech Eng. 1983; 105(3):216-25.

7. Roos H, Lauren M, Adalberth T, Roos EM, Jonsson K, Lohmander LS. Knee osteoarthritis after meniscectomy: prevalence of radiographic changes after twenty-one years, compared with matched controls. Arthritis Rheum. 1998; 41(4):687-93.

8. Mitchell J, Graham W, Best TM, Collins C, Currie DW, Comstock RD, Flanigan DC. Epidemiology of meniscal injuries in US high school athletes between 2007 and 2013. Knee Surg Sports Traumatol Arthrosc. 2016;24(3):715-22.

9. Poehling GG, Ruch DS, Chabon SJ. The landscape of meniscal injuries. Clin Sports Med. 1990:9(3):539-49.

10. Granan LP, Inacio MC, Maletis GB, Funahashi TT, Engebretsen L. Sportspecific injury pattern recorded during anterior cruciate ligament reconstruction. Am J Sports Med. 2013;41(12):2814-8.

11. Ingram JG, Fields SK, Yard EE, Comstock RD. Epidemiology of knee injuries among boys and girls in US high school athletics. Am J Sports Med. 2008; 36(6):1116-22.

12. Slauterbeck JR, Kousa P, Clifton BC, Naud S, Tourville TW, Johnson RJ, Beynnon BD. Geographic mapping of meniscus and cartilage lesions associated with anterior cruciate ligament injuries. J Bone Joint Surg Am. 2009;91(9):2094-103.

13. Dandy DJ. The arthroscopic anatomy of symptomatic meniscal lesions. J Bone Joint Surg Br. 1990;72(4):628-33.

14. Jones JC, Burks R, Owens BD, Sturdivant RX, Svoboda SJ, Cameron KL. Incidence and risk factors associated with meniscal injuries among activeduty US military service members. J Athl Train. 2012;47(1):67-73.

15. Bergkvist D, Dahlberg LE, Neuman P, Englund M. Knee arthroscopies: who gets them, what does the radiologist report, and what does the surgeon find? An evaluation from southern Sweden. Acta Orthop. 2016;87(1):12-6.

16. Shahriaree H. Meniscal injuries. In: Shahriaree H, editor. O'Connor's textbook of arthroscopic surgery. Second edition. Philadelphia: Lippincott, Inc; 1992. p. 315-21.

17. Englund M, Roos EM, Roos HP, Lohmander LS. Patient-relevant outcomes fourteen years after meniscectomy: influence of type of meniscal tear and size of resection. Rheumatology (Oxford, England). 2001;40(6):631-9.

18. Pathania VP, Kulshreshtha V, Arora NC. Arthroscopic evaluation and management of Meniscal injuries of the knee. Med J Armed Forces India. 2001;57(2):99-103.

19. Yang JH, Jeong HI, Kim TS, Park SC, Yoon JR. The management of the popliteus hiatus during lateral meniscal transplantation. Knee. 2012;19(6):959-61.

20. Staubli HU, Birrer S. The popliteus tendon and its fascicles at the popliteal hiatus: gross anatomy and functional arthroscopic evaluation with and without anterior cruciate ligament deficiency. Arthroscopy. 1990;6(3):209-20.

21. Simonian PT, Sussmann PS, van Trommel M, Wickiewicz TL, Warren RF. Popliteomeniscal fasciculi and lateral meniscal stability. Am J Sports Med. 1997;25(6):849-53.

22. Sussmann PS, Simonian PT, Wickiewicz TL, Warren RF. Development of the popliteomeniscal fasciculi in the fetal human knee joint. Arthroscopy. 2001; 17(1):14-8.

23. Kluczynski MA, Marzo JM, Rauh MA, Bernas GA, Bisson LJ. Sex-specific predictors of intra-articular injuries observed during anterior Cruciate ligament reconstruction. Orthop J Sports Med. 2015;3(2):2325967115571300.

24. Chhadia AM, Inacio MC, Maletis GB, Csintalan RP, Davis BR, Funahashi TT. Are meniscus and cartilage injuries related to time to anterior cruciate ligament reconstruction? Am J Sports Med. 2011;39(9):1894-9.

25. Kluczynski MA, Marzo JM, Bisson LJ. Factors associated with meniscal tears and chondral lesions in patients undergoing anterior cruciate ligament reconstruction: a prospective study. Am J Sports Med. 2013:41(12):2759-65.

26. Ralles S, Agel J, Obermeier M, Tompkins M. Incidence of secondary intraarticular injuries with time to anterior Cruciate ligament reconstruction. Am J Sports Med. 2015;43(6):1373-9.

27. Rath E, Richmond JC. The menisci: basic science and advances in treatment. Br J Sports Med. 2000;34(4):252-7.

28. Noyes FR, Barber-Westin SD. Treatment of meniscus tears during anterior cruciate ligament reconstruction. Arthroscopy. 2012;28(1):123-30. 
29. Hagino T, Ochiai S, Senga S, Yamashita T, Wako M, Ando T, Haro H. Meniscal tears associated with anterior cruciate ligament injury. Arch Orthop Trauma Surg. 2015;135(12):1701-6.

30. Bellabarba C, Bush-Joseph CA, Bach BR Jr. Patterns of meniscal injury in the anterior cruciate-deficient knee: a review of the literature. Am J Orthop (Belle Mead NJ). 1997;26(1):18-23.

31. Majeed H, Karuppiah S, Sigamoney KV, Geutjens G, Straw RG. All-inside meniscal repair surgery: factors affecting the outcome. J Orthop Traumatol. 2015;16(3):245-9.

32. Brambilla L, Pulici L, Carimati G, Quaglia A, Prospero E, Bait C, Morenghi E, Portinaro N, Denti M, Volpi P. Prevalence of associated lesions in anterior Cruciate ligament reconstruction: correlation with surgical timing and with patient age, sex, and body mass index. Am J Sports Med. 2015;43(12):2966-73.

33. Feucht MJ, Bigdon S, Bode G, Salzmann GM, Dovi-Akue D, Sudkamp NP, Niemeyer P. Associated tears of the lateral meniscus in anterior cruciate ligament injuries: risk factors for different tear patterns. J Orthop Surg Res. 2015;10:34.

34. Arner JW, Irvine JN, Zheng L, Gale T, Thorhauer E, Hankins M, Abebe E, Tashman S, Zhang X, Harner CD. The effects of anterior Cruciate ligament deficiency on the meniscus and Articular cartilage: a novel dynamic in vitro pilot study. Orthop J Sports Med. 2016;4(4):2325967116639895.

35. Spahn G. Arthroscopic revisions in failed meniscal surgery. Int Orthop. 2003; 27(6):378-81.

36. Borchers JR, Kaeding CC, Pedroza AD, Huston L, Spindler KP, Wright RW, Consortium M, the MG. Intra-articular findings in primary and revision anterior cruciate ligament reconstruction surgery: a comparison of the MOON and MARS study groups. Am J Sports Med. 2011;39(9):1889-93.

37. Ghodadra N, Mall NA, Karas V, Grumet RC, Kirk S, McNickle AG, Garrido CP, Cole BJ, Bach BR Jr. Articular and meniscal pathology associated with primary anterior cruciate ligament reconstruction. J Knee Surg. 2013;26(3):185-93.

38. Krutsch W, Zellner J, Baumann F, Pfeifer C, Nerlich M, Angele P. Timing of anterior cruciate ligament reconstruction within the first year after trauma and its influence on treatment of cartilage and meniscus pathology. Knee Surg Sports Traumatol Arthrosc. 2017;25(2):418-25.

39. Warren RF. Arthroscopic meniscus repair. Arthroscopy. 1985:1(3):170-2.

40. West RV. Lateral meniscal root tears associated with anterior cruciate ligament. Arthroscopy. 2004;20 Supp 1:e32-3.

\section{Submit your next manuscript to BioMed Central and we will help you at every step:}

- We accept pre-submission inquiries

- Our selector tool helps you to find the most relevant journal

- We provide round the clock customer support

- Convenient online submission

- Thorough peer review

- Inclusion in PubMed and all major indexing services

- Maximum visibility for your research

Submit your manuscript at www.biomedcentral.com/submit 